\title{
Gemeinsame Mitteilungen von BvDU und DGU
}

Urologe 2019.58:1240

https://doi.org/10.1007/s00120-019-01051-5

() Springer Medizin Verlag GmbH, ein Teil von Springer Nature 2019

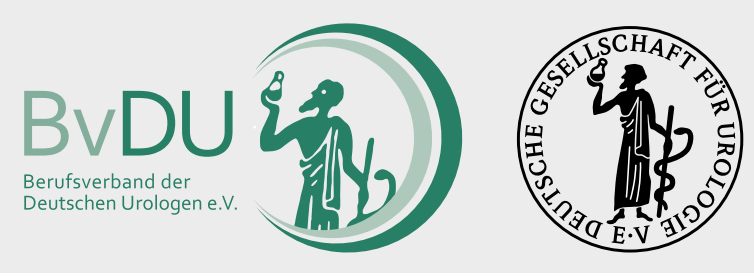

\section{Gemeinsame Erklärung}

\author{
zur Zusammenarbeit der urologischen Vereine \\ Deutsche Gesellschaft für Urologie e.V. und \\ Berufsverband der Deutschen Urologen e.V.
}

\begin{abstract}
Am 23. August 2019 fand auf Einladung des DGU-Generalsekretärs in Berlin eine gemeinsame Konferenz zur zukünftigen Ausrichtung und Zusammenarbeit der beiden Vereine statt. Im Ergebnis der Konferenz bestand Einvernehmen, dass es sich bei den Vereinen um eigenständig agierende Vereinigungen handelt, die partnerschaftlich mit einander kommunizieren, gleichwohl aber unterschiedliche Kompetenzbereiche aufweisen, wobei es in Teilbereichen durchaus zu Überschneidungen und Kompetition kommen kann. Die Fachgesellschaft und der Berufsverband sind sich einig und überzeugt, dass ein konstruktiver Dialog zwischen den beiden Vereinen wichtige und notwendige Basis für die erfolgreiche Vertretung der Interessen des Fachgebietes Urologie gegenüber allgemeiner Öffentlichkeit, Gesundheitspolitik und Selbstverwaltung ist.
\end{abstract}

Für beide Vereine steht die zukunftsorientierte urologische Patientenversorgung im Mittelpunkt des jeweiligen Wirkens. Für die Fachgesellschaft liegt der Schwerpunkt im Bereich Forschung, Lehre, Fortbildung, Patienteninformation, Weiterentwicklung und medizinische Positionierung des Faches sowie der Krankenversorgung. Der Berufsverband fokussiert in seiner Arbeit auf die standes- und berufspolitische Interessenvertretung der Urologie mit besonderem Blick auf die ärztliche Freiberuflichkeit und unternehmerische Selbstständigkeit.

Ausgehend von diesem Grundverständnis der Vereinsaufgaben verständigen sich beide Seiten auf eine offen-konstruktive und vertrauensvolle Zusammenarbeit und unterstützen sich wechselseitig im Rahmen ihrer satzungsgemäßen Arbeit jeweils aktiv in den jeweiligen Kompetenzbereichen. Grundlage für diese vertrauensvolle $\mathrm{Zu}$ sammenarbeit ist ein kontinuierlicher Informations- und Erfahrungsaustausch zwischen dem DGU-Vorstand und dem BvDU-Präsidium im Rahmen gemeinsamer Sitzungen. Das Nähere regelt eine noch zu schließende Rahmenvereinbarung. Fachgesellschaft und Berufsverband werden in regelmäßigen Abständen die Struktur und Ergebnisse ihrer Zusammenarbeit evaluieren und auf dieser Basis konstruktiv weiterentwickeln.

Auch zukünftig werden beide Vereine im intensiven Dialog gemeinsame Projekte definieren und deren Umsetzung gestalten. Insbesondere die bevorstehenden strukturellen Veränderungen im Gesundheitssystem stellen für die Zukunft des Faches Urologie eine Herausforderung dar. Fachgesellschaft und Berufsverband sind sich der Bedeutung dieses Veränderungsprozesses bewusst und streben eine intensive Zusammenarbeit an, um entsprechende eigene Impulse für die Urologie in diesem Prozess setzen zu können.
Bei kompetitiven Projekten an der Schnittstelle der Kompetenzbereiche sind sich die Vereine der gemeinsamen Verantwortung für die allgemeine Öffentlichkeit und die urologische Gemeinschaft bewusst. Beide Seiten verstehen Kompetition daher als inspirierend und als wechselseitigen Ansporn für die Weiterentwicklung der urologischen Patientenversorgung. Die gemeinsame Geschichte und die jahrzehntelange konstruktive Zusammenarbeit der Vereine bilden die Grundlage der gegenseitigen Wertschätzung und des Umgangs.

Um die Wahrnehmung der gesundheitspolitischen sowie wissenschaftlichen Botschaften und Positionen der beiden Vereine, insbesondere in ihrer Außenwirkung, zu stärken und -sofern möglich- zielführend zu synchronisieren, wird die seit Jahren in der gemeinsamen „Kommission Öffentlichkeitsarbeit" praktizierte Koordination der Öffentlichkeitsarbeit fortgesetzt. Darüber hinaus werden sich beide Seiten in ihrer eigenständigen Öffentlichkeitsarbeit im Bereich ihrer Kompetenzbereiche kontinuierlich austauschen und den jeweiligen anderen Verein frühzeitig informiert halten sowie anlass- und themenbezogen auch gemeinsame Positionspapiere oder Pressemitteilungen herausgeben.

Berlin im August 2019

\section{Prof. Dr. med. Maurice Stephan Michel}

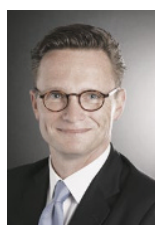

Generalsekretär der Fachgesellschaft

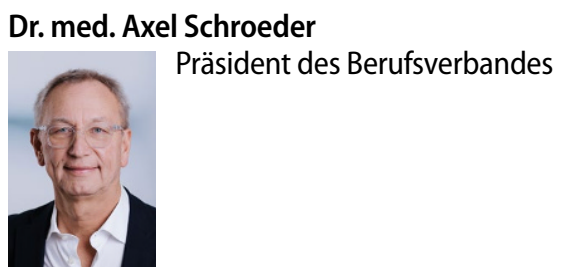

